Cognitive neuropsychology

\section{Sensation and semantics}

\section{John C. Marshall}

"NIни est in intellectu quod non primus in sensu" (Nothing is understood by the intellect which is not first perceived by the senses) - thus wrote Aristotle some 2,000 years ago. The motto has stood the test of time pretty well, despite some chipping away by those who argue that the innate organization of the central nervous system imposes severe constraints upon the mind's interpretation of sensation ${ }^{1,2}$

Nonetheless, it would be quite rational to suppose that although thoughts must be triggered by experience, the conceptual and encyclopaedic knowledge so accessed is coded in a form independent of sensory modality. Suppose I believe pigs to be "unclean, medium-sized quasi-domestic animals providing pork and bacon for the table". Surely I access that neurologically unitary knowledge, irrespective of whether I see, smell or hear a pig, or see or hear the word pig? To suggest duplication of such knowledge - that is, an identical or at least highly similar body of information is stored once in conjunction with the look of pigs and once again with the sound of pig - seems decidedly counter to common sense. And yet, if the interpretation that McCarthy and Warrington ${ }^{3}$ place upon their results, reported on page 428 of this issue, is correct, common sense would appear to be wrong.

It is now reasonably well established that acquired brain damage can selectively impair knowledge within specific conceptual categories ${ }^{4}$. McCarthy and Warrington's patient (T.O.B.) sustained relatively focal damage to the left temporal lobe that resulted in a selective inability to define the characteristics of animals or plants in response to their spoken names; by contrast, he is consistently much better at providing information about inanimate objects. Thus in response to 'pig', T.O.B. could only volunteer that it was an animal, whereas 'wheelbarrow' elicited the reply, "the item we have here in the gardens for carrying bits and pieces; wheelbarrows are used by people doing maintenance here on your buildings. They can put their cement and all sorts of things in it to carry it around". So far so good: comparable phenomena of category-specific impairment have now been widely reported in cases of both acquired $^{5}$ and developmental ${ }^{6}$ pathology.

But the performance of T.O.B. adds a further puzzle to the pattern. Although he is unable to define adequately the spoken names of living things, T.O.B. succeeded in providing the relevant details when given a picture to identify. In response to the word 'rhinoceros', he can only manage, "Animal, can't give you any functions"; yet a picture thereof elicits the information "Enormous, weighs over one ton, lives in Africa". From this reliable in conjunction with pictures of whales; but that I later learned verbally that 'whales', by virtue of their biology, are mammals not fish. This developmental history could accordingly programme two neuronal substrates, in the visual association cortex and auditory association cortex, with different pieces of information about the same referent. If there was mutual transfer of information between these loci, duplicate encyclopaedias could develop, each accessed by a particular sensory modality. In principle, acquired brain damage could thus destroy one information store whilst leaving the other relatively intact. The upshot would be a modality-specific loss of knowledge,

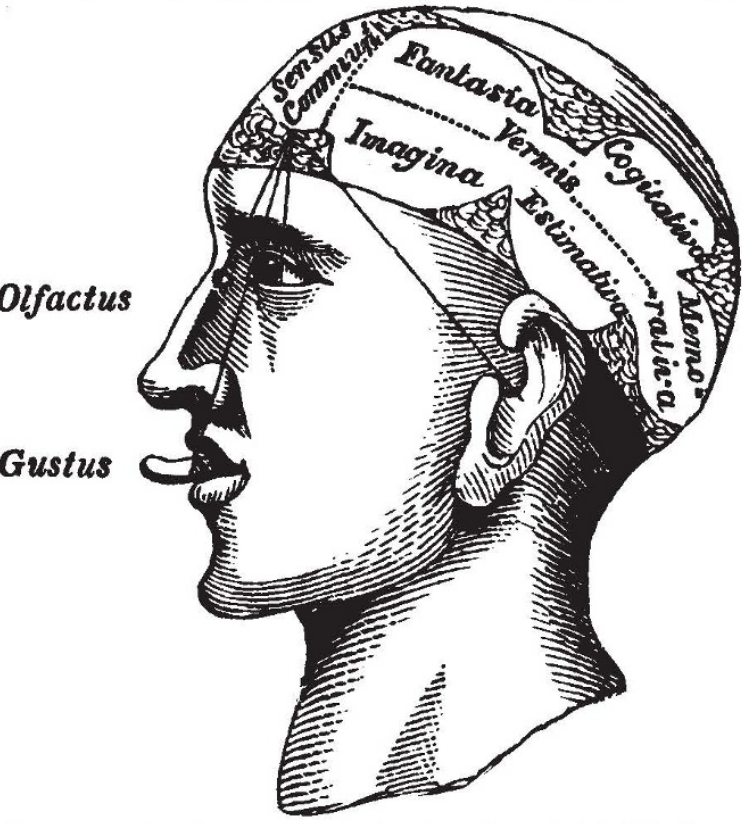

Our expression 'common sense' arises from Aristotle's theory that there is a general sense common to all the special senses. The communis sensus is seen at the front of the brain in this illustration, first published in a translation of J. F. Blumenbach's Institutiones physiologicae, published in 1840. This is, in fact, he location of the frontal cortex, now known to be involved in integrating information from the various senses. (From ref. 9.) exactly the result that McCarthy and Warrington report ${ }^{3}$.

A similar dissociation could occur without duplication of information. Suppose that knowledge acquired in conjunction with a picture was stored uniquely in visual association cortex, and that knowledge acquired in conjunction with the name of the picture's referent was stored only in language-committed cortex. Bidirectional associative links between the two sets of information could then enable the normal brain to retrieve the full corpus of knowledge, irrespective of whether the system is addressed through picture or word. But if these linkages were broken, presentation of word or picture would only access the knowledge originally acquired in each respective condition.

On this hypothesis, the brain could store contradictory information about the same referent without consciously bringing the anomaly to mind. This might seem like a reductio ad absurdum of the notion of modality-specific cognitive deficits. But before we dismiss

pattern of performance, McCarthy and Warrington draw the conclusion that semantic information is multiply represented in the normal brain and that this duplication is linked to the input modalities whereby we gain knowledge of the world. (For purposes of the argument, language must be viewed as a discrete input system, analogous in this regard to the senses ${ }^{7}$.) The conclusion that T.O.B. has sustained modality-specific loss of information about living things, rather than differential impairment of access to such knowledge, is reinforced by the observation that he is highly consistent with respect to the particular items on which he fails.

McCarthy and Warrington support their hypothesis with an ontogenetic argument. Let us suppose, not unreasonably, that I first acquired the knowledge that whales are large sea-living creatures the idea, we should recall a patient (A.R.) reported by Warrington and Shallice ${ }^{8}$. To the auditory stimulus 'Solzhenitzyn', A.R. responded that this was the name of a living Russian novelist. But the written stimulus Solzhenitzyn he claimed was the name of a Polish novelist who died about 1900. Our understanding of the brain still lies in the heart of darkness.

\footnotetext{
1. Leibniz, G.W. New Essays on Human Understandin (Cambridge University Press, 1981)

2. Chomsky, N. Knowledge of Language (Praeger, New York, 1986)

3. McCarthy, R. A. \& Warrington, E.K. Nature 334, 428-430 (1988)

4. Shallice, T. Cognitive Neuropsych. 5, 133-142 (1988). 5. Warrington, E.K. \& Shallice, T. Brain 107, 829-854 (1984). Temple, C.M. Brain 109, 1225-1242 (1986).

Marshall J.C. Cognition 17, 209-242 (1984).

Warrington, E. K \& Shallice. T. Brain 102, 43-63 (1979)

9. Clarke, E. \& Dewhurst, K. An Illustrated History of Brain Function (Sandford, Oxford, 1972).
}

John C. Marshall is in the Neuropsychology Unit, Department of Clinical Neurology, Radcliffe Infirmary, Oxford $O X 26 H E, U K$. 